\title{
Automatic Tariff Calculation with Wireless Energy Meter
}

\author{
S.Rama Devi ${ }^{1}$, C.Srujana ${ }^{2}$ \\ Department of ECE, AUCEW, Visakhapatnam, A.P, India ${ }^{1,2}$
}

\begin{abstract}
The existing energy meter reading technology uses man power which has disadvantages like errors during calculation, absence of consumer during billing time and extra expenses for the billing process. The wireless energy meter monitoring system aims to minimize these difficulties by providing energy meter monitoring through wireless medium. Power consumed by consumer in the home side module is monitored by Electricity Board through wireless technology. This wireless technology is implemented by using ZigBee modules. This is done by using a ZigBee transceiver interfaced with both the EB (Electricity Board) section server as well as the consumer side. The Microcontroller based system continuously records the readings. These live meter readings are displayed in the LCD and also sent to the EB side through ZigBee. The EB (Electricity Board) takes the responsibility of calculating the bill with the data received from the home side, and the tariff provided by the operator and displays the same in the LCD placed in the consumer's home by transmitting the data through ZigBee transceiver. If at all the consumer doesn't pay the bill in allotted time, then the power supply to the home is cut down automatically using relay.
\end{abstract}

Keywords: Zigbee module, LCD, EB module.

\section{I .INTRODUCTION}

Energy meter is a device that measures the amount of energy supplied to or produced from a residence or building. The electromechanical induction meter operates by counting the revolutions of a non-magnetic, but electrically conductive, metal disc which is made to rotate at a speed proportional to the power passing through the meter making the number of revolutions proportional to energy. Electronic meters display the energy used on an LCD or LED display, and some can also transmit readings to remote places. Electronic meters can also record other parameters of the load and supply such as instantaneous and maximum rate of usage demands, voltages, power factor, etc. They can also support time-of-day billing, i.e. recording the amount of energy used during on-peak and off-peak hours. Taking energy meter reading is time consuming and an expensive task. Meter reader should travel long distances to take reading manually, consumers should stand in a long line and submit the bill. Existing automatic meter reading technology uses manpower which has disadvantages like errors during calculation, absence of consumer during billing time and extra expenses for the billing process.

The wireless technology is implemented by having a ZigBee transceiver interfaced with the EB (Electricity Board) section server as well as in the consumer side. The units are sensed in the consumer side and sent to EB (Electricity Board) section through ZigBee module and they are displayed in the LCD (Liquid Crystal Display) and GUI (Graphical User Interface). The calculation of tariff is done in EB (Electricity Board) section and sent to consumer side through ZigBee module which is displayed in the LCD and GUI (Graphical User Interface). This process goes on for a month and after a month the total amount to be paid is displayed in the LCD (Liquid Crystal Display) and the sensing of units for the next month is started.
If the consumer pays the bill in the allotted time then the total amount to be paid is displayed as 0 . If at all the consumer does not pay the bill in the allotted time then the power supply is cut to the home of consumer.

\section{DESCRIPTION}

The existing energy meter reading technology uses manpower which has many disadvantages like errors during calculation, absence of consumer during billing time and extra expenses for the billing process. The wireless energy meter monitoring system aims to minimize these difficulties by providing automatic energy monitoring through wireless medium. The wireless technology is implemented by using a ZigBee transceiver interfaced with both the EB (Electricity Board) section server as well as in the consumer side. The IR sensor is fixed in the energy meter which senses the number of units consumed and displays the units in the LCD (Liquid Crystal Display). These units are transmitted through ZigBee to the GUI (Graphical User Interface) in EB (Electricity Board) section. The cost is calculated and displayed in the GUI (Graphical User Interface) and also it is transmitted back to the consumer's home using ZigBee which is displayed in the LCD (Liquid Crystal Display) present in consumer's home. After a fixed amount of time, the tariff for the consumed units during that fixed amount of time is displayed in both LCD (Liquid Crystal Display) and GUI (Graphical User Interface). If the consumer does not pay the bill within the allotted period of time the power is cut down using a relay, else the power supply is given without any interruption.

\section{Energy meter}

The energy meter is an electrical measuring device, which is used to record electrical energy consumed in terms of units. 


\section{BLOCK DIAGRAM}

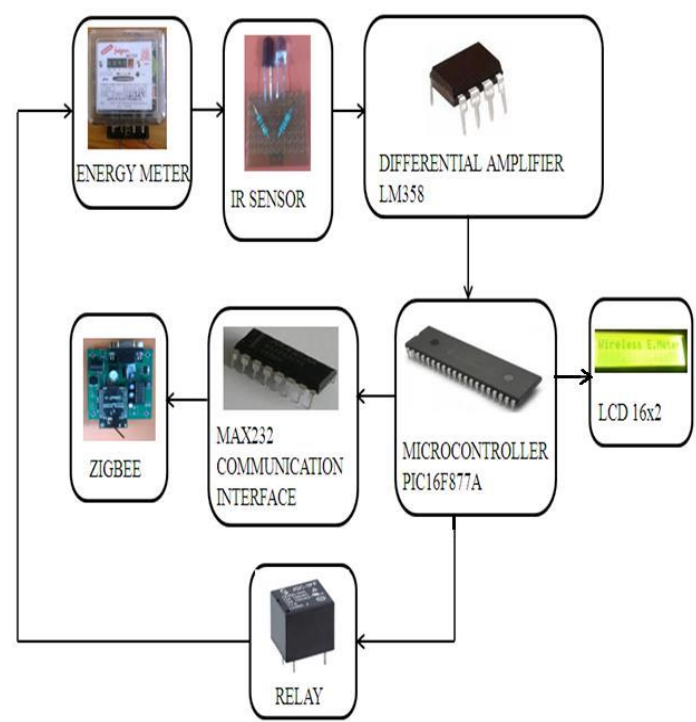

Fig1 Block diagram of consumer module

\section{IR sensor}

Infra Red (IR) sensor is an electronic sensor that measures Infra Red (IR) radiating from objects in its field of view. It is connected inside the energy meter to sense the rotations in the disk and count the number of units consumed.

\section{Differential amplifier}

LM358 is the differential amplifier used in this project. The output of the sensor is low. So in order to amplify the output, LM358 is used.

\section{Micro controller}

PIC16F877A is the micro controller used in this project for monitoring the energy meter readings.

\section{Communication interface}

MAX232 is used as communication interface to send and receive signals given by the micro controller. It converts the data into serial manner and sends to the ZigBee transceiver.

\section{ZigBee}

ZigBee is a wireless network for home and building automations. In this project ZigBee is attached with both consumer side and EB (Electricity Board) side. The sensed units in home side module are sent to the EB side through this ZigBee and at EB side the tariff calculation is done and the amount to be paid is sent to the home side through this ZigBee transceiver which is displayed in the LCD.

\section{LCD}

Liquid Crystal Displays (LCD) are used to display numeric and alpha numeric data. In this project a $16 * 2$ LCD is used to display the number of units consumed in the home side, their corresponding cost and also the amount to be paid.

\section{Relay}

A relay is an electrical switch that opens and closes under the control of another electrical circuit. In this project a relay is used to trip the power supply in case the bill is not paid in given time.

\section{Block diagram of EB (Electricity Board) Module}

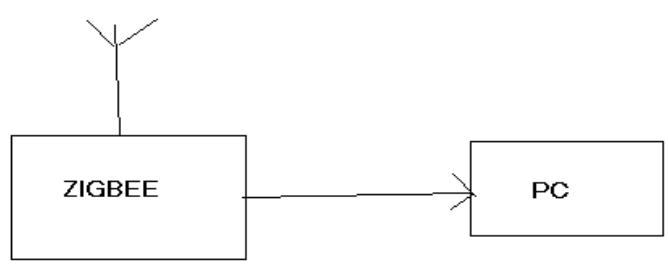

Fig: 2 Block diagram of EB module

The consumer side module consists of a ZigBee transceiver interfaced with a personal computer having Graphical User Interface (GUI). The ZigBee transceiver receives the sensed units from consumer's home and displays them on GUI. Tariff calculation is done here and the amount to be paid for the consumed units is transmitted to the consumer's home through ZigBee transceiver.

\section{III .FLOW CHART}

Flow chart of wireless energy meter monitoring system with automatic tariff calculation

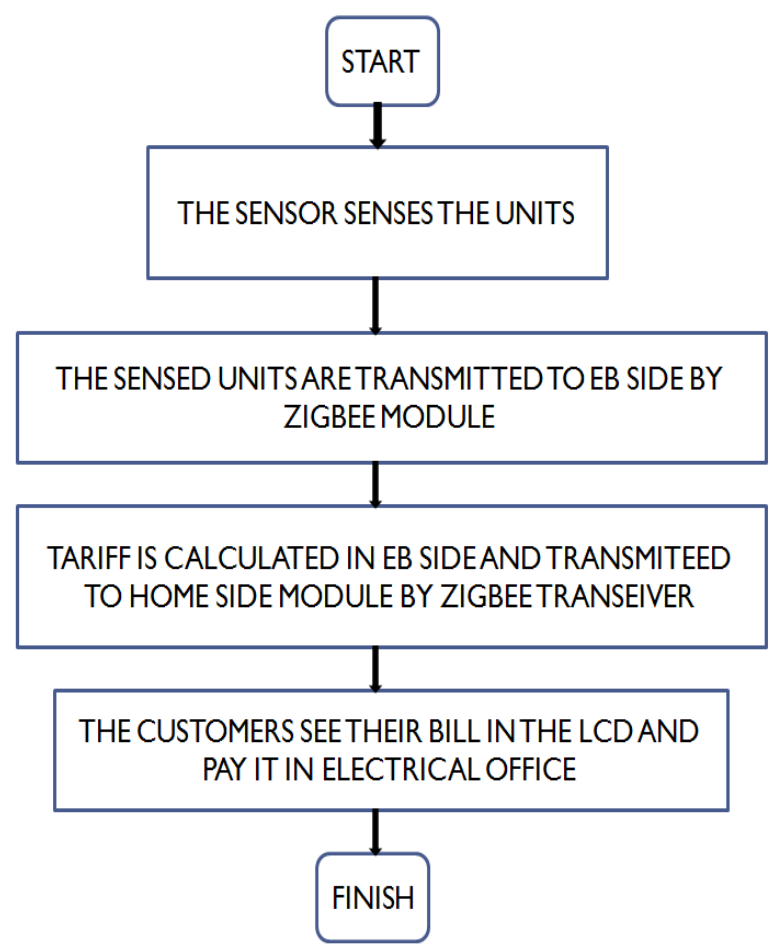

Fig: 3.1 flow chart of automatic tariff calculation with wireless energy meter

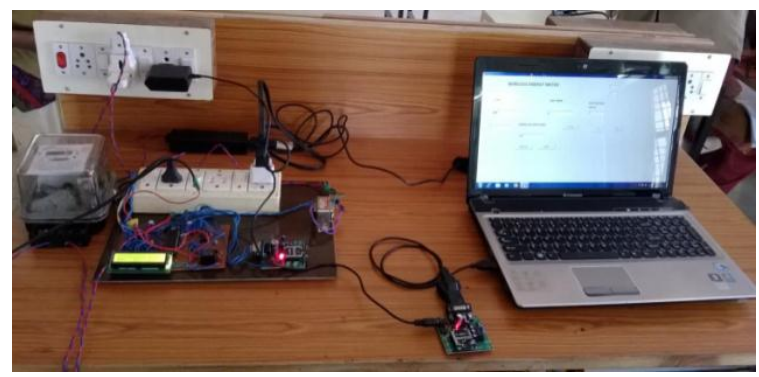

Fig 3.2: Final Prototype 


\section{IV .OUTPUTS ON LCD AND GUI}

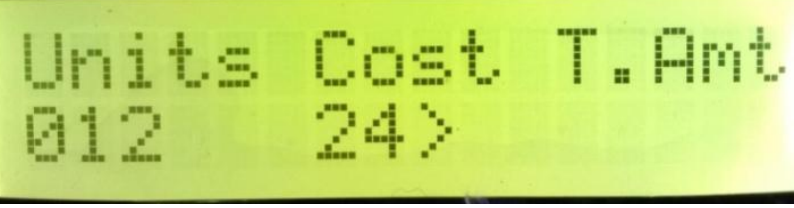

Fig 4.1: LCD displaying units and cost

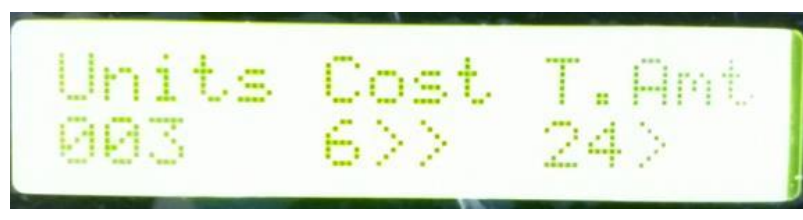

Fig: 4.2: LCD displaying units and cost of present month

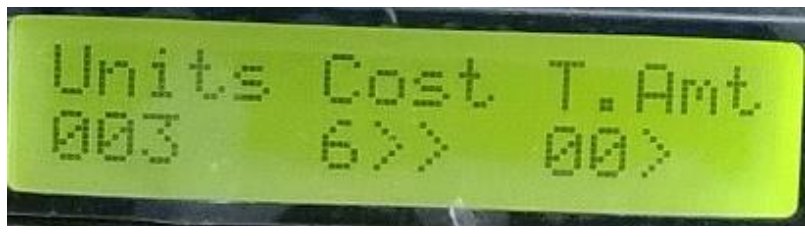

Fig.4.3 LCD display showing units and cost of the present month and that amount is paid

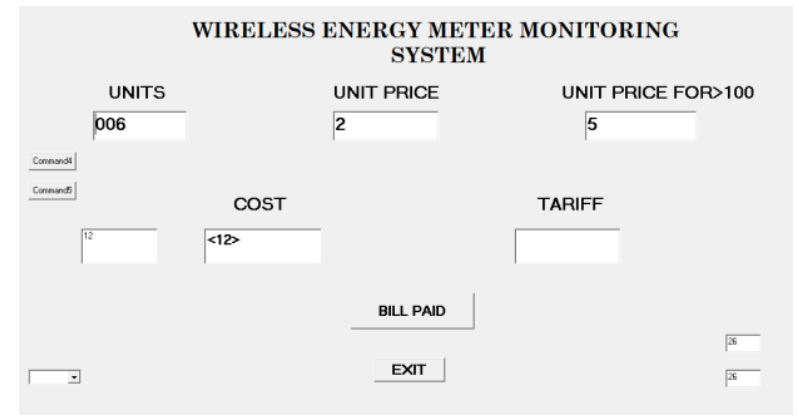

Fig.4.4 GUI displaying units and cost

\section{CONCLUSION}

Wireless energy meter monitoring system with automatic tariff calculation has been designed and tested successfully. This reduces the work of the office person to a great extent. The wireless energy meter monitoring system also reduces the difficulty faced by the people when readings are taken manually. The wireless energy meter monitoring system has been further extended by adding an additional feature of tripping the supply to a particular customer in case bill is not paid. The tariff changes can also be easily updated by the Electricity Board when needed. The user can see the units and cost in the LCD and can control the usage of electricity.

\section{REFERENCES}

1. Automatic energy calculation through wireless smart meter using ZigBee in International Journal of Computer Science and Communication Networks by Vignesh Setal

2. Visual Basic 6.0 Made Easy by Liew Voon Kiong. 